\section{Forscher finden den G-Punkt nicht}

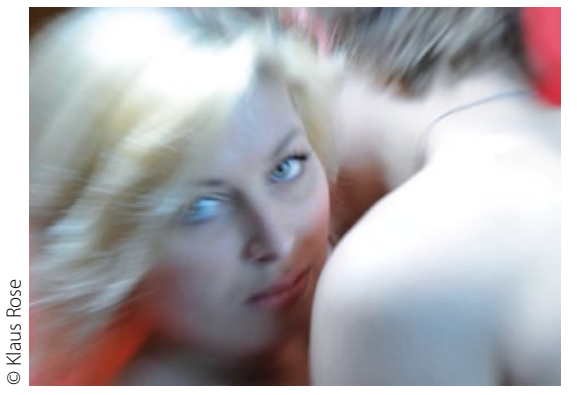

Beim Sex hört für zwei Wissenschaftler der Spaß auf. G-Punkt? „Gibt's nicht“, sagen sie.

\section{Mehr als $\mathbf{3 0 0}$ Millionen Treffer erhält, wer den Begriff "G-Spot" in Google eintippt. Im Angebot sind Anlei- tungen, wie der G-Punkt zu finden und zu stimulieren wäre, ebenso wie Einladungen, ihn zur höheren sexuellen Erbauung kollagengestützt vergrößern zu lassen. Aber existiert er überhaupt?}

- Benannt ist der G-Punkt nach Ernst Gräfenberg, einem 1957 verstorbenen Gynäkologen. Gräfenberg selbst hat weder den Ausdruck gebraucht, noch eine entsprechende Struktur postuliert. In einem Aufsatz von 1950 befasste er sich lediglich mit der Rolle der Urethra beim weiblichen Orgasmus (Int J Sexol 1950; 3: 145-148). Die Rede vom G-Punkt kam erst Anfang der 1980er-Jahre in den USA auf. Mittlerweile hängen von dem mythischen Ort der Ekstase in der Vorderwand der Vagina entlang der Harnröhre buchstäblich Millionen $\mathrm{ab}$ - Millionen Dollar nämlich, die weltweit von Frauen etwa in die Vergrößerung ihres G-Punktes, auch Augmentation genannt, investiert werden.

Dass dieses Geld gut angelegt ist, bezweifeln Vincenzo Puppo vom Centro Italiano di Sessologia und der israelische Neurourologe Ilan Gruenwald. Die beiden haben sich die Literatur zum Thema angesehen und kommen zu dem Schluss: „Die veröffentlichten wissenschaftlichen Fakten deuten darauf hin, dass der Gräfenberg-Punkt nicht existiert." Speziell kritisieren sie eine kürzlich publizierte Studie von Adam
Ostrzenski vom Institute of Gynecology (St. Petersburg/Florida), der den G-Punkt bei einer 83-Jährigen anatomisch lokalisiert haben will - ein einseitiges Vergnügen, da die Frau auf dem Seziertisch gelegen hatte (J Sex Med 2012; 9: 1355-9). Histologische Ergebnisse habe Ostrzenski nicht publiziert, monieren Puppo und Gruenwald. „Es scheint völlig unangemessen zu behaupten, die Existenz des G-Punktes sei nun dokumentiert. Diese Behauptung basiert auf einer einzigen Sektion, und zwar durch einen Arzt, der an einem kommerziell orientierten Institut beteiligt ist." Tatsächlich nimmt Ostrzenski am Institute of Gynecology allerlei kosmetisch-gynäkologische Eingriffe vor, darunter auch G-Punkt-Plastiken. Mit seiner "neuen anatomische Entdeckung des G-Punktes" wird auf den Internetseiten des Instituts geworben (http://cosmetic-gyn.com/).

Mit ihrer Ansicht, die den G-Punkt ins Reich der Mythen verweist, stehen Puppo und Gruenwald nicht allein. Der Bioethiker Jeffrey Spike vom Florida State University's College of Medicine hält die Geschichte vom G-Punkt für ein „Volksmärchen“. Und der New Yorker Neurowissenschaftler Terence Hines von der Pace University nennt den G-Punkt ein "gynäkologisches UFO: viel gesucht, viel diskutiert, aber objektiv nicht verifiziert". Ein Bereich wie der G-Punkt müsse reich innerviert sein; eine solche Struktur habe noch niemand histologisch nachgewiesen.

Ungeachtet seiner dubiosen Existenz hat der G-Punkt in den vergangenen Jahren einige Geschwister bekommen, etwa den APunkt (am anterioren Fornix), den U-Punkt (Urethra) oder den K-Punkt (hier schwanken die Ortsangaben). Gräfenberg selbst hatte in seinem Aufsatz übrigens von „unzähligen erotogenen Stellen“ geschrieben: „Wir können beinahe sagen, es gibt keinen Teil des weiblichen Körpers, der keine sexuelle Reaktion zeigen würde." Für Ärzte, die sich auf kollagenöse Augmentation spezialisiert haben, bleibt also noch einiges zu tun.

DR. ROBERT BUbLAK -

- Puppo V, Gruenwald I. Int Urogynecol J 2012; DOI: 10.1007/s00192-012-1831-y

\section{Ist Oralsex karzinogen?}

Ca. 18000 Kopf-Hals-Tumoren werden in Deutschland pro Jahr neu diagnostiziert, Tendenz steigend. Hinter jedem zweiten Tumor im Rachenraum und hinter jedem vierten Tumor der Mundhöhle steckt wahrscheinlich eine HPV-Infektion. Übertragen weden die Humanen Papillomaviren beim Oralsex. Nach einer norwegischen Studie nimmt die Inzidenz der HPV-assoziierten Tumoren im Mund-Rachen-Raum um 5-6\% pro Jahr zu.

- Vortrag im Rahmen der 83. Jahresversammlung der Deutschen Gesellschaft für Hals-NasenOhren-Heilkunde, Kopf- und Hals-Chirurgie e.V., Bonn, 16.-20.5.2012

\section{Therapieresistenter Tripper}

Die Weltgesundheitsorganisation WHO warnt vor der zunehmenden Verbreitung antibiotikaresistenter Gonokkoken. U. a. in Frankreich, Japan, Australien, Schweden, Norwegen und Großbritannien wurden Infektionen mit multiresistenten Neisseria gonorrhoeae beobachtet.

- http://www.who.int/mediacentre/news/ notes/2012/gonorrhoea_20120606/en/

Neue Patientenbroschüre zu funktionellen Herzbeschwerden Patienten mit funktionellen Herzbeschwerden haben oft viele Fragen - beispielsweise, ob nicht doch eine ernste Herzerkrankung hinter ihren Beschwerden steckt. Die kostenlose Patientenbroschüre "20 Fragen - 20 Antworten" zum Thema "Herzstolpern und Brustschmerzen" soll hier aufklären. Sie können schon vorab ein Paket mit 50 Exemplaren der Broschüre (plus einem Exemplar einer zusätzlichen Arztinformation über einen ganzheitlichen Therapieansatz) fürs Wartezimmer bestellen unter vertrieb@springer.com oder per Fax an die Nr. 061 02/50 62 40; Stichwort "20 Fragen - Herzstolpern und Brustschmerzen".

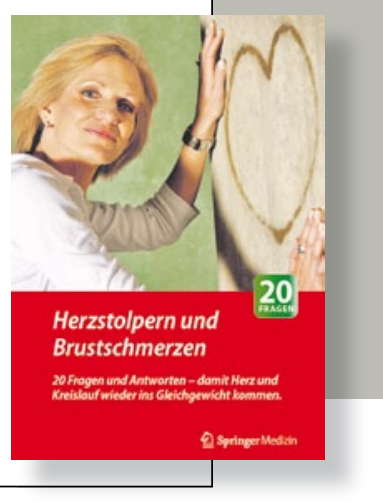

